\title{
Precisión diagnóstica de la ecografía en el diagnóstico de cáncer diferenciado de tiroides
}

\section{Diagnostic accuracy of ultrasonography for diagnosing differentiated thyroid cancer}

\author{
Romina A. Gecchelin ${ }^{1}$, María C. Pereyra ${ }^{1}$, Mauro J. Pautasso ${ }^{1}$, Constanza F. Ramacciotti ${ }^{1}$, María L. \\ Bertolino $^{1}$, Analía V. Checa ${ }^{1}$, Eduardo N. Cohen ${ }^{1}$. \\ Correspondencia: Gecchelin Romina Alejandra. Servicio de Endocrinología. Clínica Universitaria Reina Fabiola Oncativo 1248-X5004FHP-Cordoba, Argentina; \\ email: rogecchelin@gmail.com
}

\section{Resumen}

INTRODUCCIÓN: El carcinoma diferenciado de tiroides es la neoplasia endócrina más prevalente. En Argentina representa el 2,2\% de los cánceres en mujeres y en los hombres corresponde al $0,5 \%$. La ecografía es el método inicial de screening de los nódulos tiroideos y la citología por aspiración con aguja fina (PAAF) es la prueba diagnóstica confirmatoria de elección. Sin embargo, existe una limitación debido a la variabilidad intra e interobservador en los estudios de imágenes y citopatológico.

OBJETIVO: Determinar la precisión diagnóstica de los criterios ultrasonográficos sospechosos de malignidad de nódulos tiroideos para el diagnóstico de cáncer de tiroides. Determinar el valor de corte del número de criterios ecográficos de sospecha de malignidad que muestre el mejor balance de sensibilidad/especificidad para diagnosticar cáncer de tiroides.

PACIENTES Y MÉTODOS: Estudio observacional, retrospectivo, de pruebas diagnósticas. Se incluyeron pacientes adultos con nódulos sospechosos de malignidad por ecografía y punción aspirativa. Se analizaron las historias clínicas de pacientes de ambos sexos intervenidos mediante cirugía tiroidea entre el 1 de enero de 2012 y el 31 de diciembre de 2018 en la Clínica Universitaria Reina Fabiola en la Ciudad de Córdoba. Se evaluó la sensibilidad, especificidad, valores predictivos positivo y negativo de la presencia de al menos un criterio ecográfico positivo para malignidad tomando como gold standard el resultado anatomopatológico. Se analizó el valor de corte del número de criterios ecográficos que mostrara la mejor relación de sensibilidad/especificidad.

RESULTADOS: Se incluyeron 223 pacientes (56\%). De ellos $179(80 \%)$ eran de sexo femenino, con una edad media (desviación estándar - DE) de 43,49 (13,03) años. Entre los pacientes con patología benigna, 65 casos (52\%) resultaron en adenoma folicular (hallazgo más frecuente). Noventa y nueve (44\%) pacientes presentaron diagnóstico de carcinoma diferenciado de tiroides (CDT). Setenta y seis (76.81\%) eran de sexo femenino con una edad media (DE) de 40,36 (12,48) años. La media (DE) del tamaño tumoral fue 20,35 $(12,73) \mathrm{mm}$. El 94,45\% resultaron CDT papilar cuya variante más frecuente fue la clásica con 72 de 94 (73\%) casos. La media (DE) de criterios ecográficos sospechosos en el grupo de pacientes con malignidad fue de 2,10 (1,22) y para el grupo de pacientes con resultado benigno fue de $1,36(1,08)(\mathrm{p}<0,001)$. La sensibilidad de los criterios ecográficos sospechosos de malignidad para el diagnóstico de CDT fue superior en el criterio hipoecogénico con el 70,7\%, y la especificidad fue alta en los siguientes criterios: más alto que ancho, microcalcificaciones, bordes irregulares, invasión perinodular y adenopatías. El valor de corte 
del número de criterios ecográficos sospechosos de malignidad que mostró el mejor balance de sensibilidad/especificidad para diagnosticar cáncer de tiroides.

CONCLUSIONES: La ecografía es el método de screening inicial de los nódulos tiroideos. Si bien algunos criterios ecográficos tienen mayor sensibilidad que otros para el diagnóstico de cáncer de tiroides, la precisión diagnóstica del método se incrementa a medida que aumenta el número de características sospechosas.

Palabras claves: cáncer de tiroides, ultrasonografía, nódulo tiroideo.

\section{Abstract}

INTRODUCTION: Differentiated thyroid carcinoma is the most prevalent endocrine neoplasm. In Argentina, it represents $2.2 \%$ of all cancers that occur annually in women while in men it corresponds to $0.5 \%$ of the total cases detected. Ultrasound is the initial screening method for thyroid nodules and fine needle aspiration cytology (FNA) is the confirmatory test for the most appropriate diagnosis and treatment. However, there is a limitation due to intra and interobserver variability in imaging and cytopathological studies.

OBJECTIVES: To determine the diagnostic accuracy of the ultrasonographic features for the diagnosis od thyroid nodules malignancy. To determine the cut-off value of the number of ultrasound suspicious criteria for malignancy that shows the best balance of sensitivity / specificity for diagnosing thyroid carcinoma.

PATIENTS AND METHOD: This is an observational, retrospective, diagnostic test study that included adult patients with ultrasound and aspiration punction of nodules suspicious of malignancy. The sensitivity, specificity, positive and negative predictive values of the presence of at least one positive ultrasound criterion for malignancy were evaluated, taking the histopathology results as a gold standard. Likewise, it analyzed the cut-off value of the number of ultrasound criteria that shows the best sensitivity / specification ratio.

RESULTS: Data from 223 patients (56\%) were included. Of these, $179(81 \%)$ were female, with a mean age (standard deviation - SD) of 43.49 (13.03) years. Among patients with benign pathology, $65(52 \%)$ cases resulted in follicular adenoma (most frequent pathological finding). Ninety nine (45\%) patients had a diagnosis of differentiated thyroid carcinoma. Seventy six (77\%) were female with a mean age (SD) of 40.36 (12.48) years. The mean (SD) of the tumor size was 20.35 (13) $\mathrm{mm}$. Ninety five percent were papillary CDT whose most frequent variant was the classic one with 72 of 94 (73\%) cases. The mean (SD) of suspicious ultrasound criteria in the group of patients with malignancy was 2.10 (1.22), for the group of patients with benign results it was $1.36(1.08)(\mathrm{p}<0.001)$. The sensitivity of the ultrasonographic criteria suspected for malignancy for the diagnosis of differentiated thyroid cancer was higher for the hypoechogenic criterion (0.7) and the specificity was higher in the following criteria: taller than wide, microcalcifications, irregular borders, perinodular invasion and adenopathies. The cut-off value of the number of ultrasonographic criteria suspicious of malignancy that showed the best sensitivity / specificity balance for diagnosing thyroid cancer calculated was 2 .

CONCLUSION: Ultrasound is the initial screening method for thyroid nodules. Although some ultrasound criteria are more sensitive for the diagnosis of thyroid cancer than others, the diagnostic accuracy of the method increases as the number of suspicious features detected increases.

Keywords: Thyroid neoplasms, ultrasonography, thyroid nodules.

\section{Introducción}

Los nódulos tiroideos afectan a uno de cada 14 individuos en la población general y aproximadamente el 5\% corresponden a tumores malignos de la glándula tiroides ${ }^{1}$.

El carcinoma diferenciado de tiroides (CDT) (carcinoma papilar, carcinoma folicular y variantes) es la neoplasia originada en glándulas endocrinas más prevalente, y deriva de la degeneración maligna de las células foliculares tiroideas. Representa a nivel mundial el $1 \%$ de todas las neoplasias y, de todos los tumores malignos de tiroides, el CDT abarca un $80-90 \%$ de los casos? ${ }^{2}$.

En Argentina representa el 2,2\% de todos los cánceres que ocurren anualmente en mujeres, ubicando al cáncer de tiroides en el décimo lugar. En los hombres, corresponde apenas al 0,5\% del total de casos detectados, ocupando el vigésimo lugar ${ }^{3}$. 
El CDT afecta principalmente al sexo femenino (60-80\%), con mayor frecuencia entre los 30 y 60 años.

El número de casos diagnosticados se ha incrementado notablemente en la última década debido al mayor uso de los métodos complementarios de diagnóstico por imágenes. Sin embargo, a pesar del aumento en la incidencia, la mortalidad se mantuvo estable a lo largo de las últimas décadas ${ }^{2}$

En la mayoría de casos la enfermedad está confinada en la región cervical en el momento del diagnóstico, con un alto potencial curativo luego de la intervención quirúrgica ${ }^{4-5}$.

La ecografía es el método inicial de screening de los nódulos tiroideos con una sensibilidad reportada del $88 \%{ }^{6}$. Se estima que su utilización podría demostrar su presencia hasta en un $67 \%$ de la población, de los que serían malignos aproximadamente el 5\%1. Tiene un papel imprescindible en la evaluación de la tiroides, pues permite determinar la presencia de nódulos (únicos o múltiples) y las características de los mismos. Los hallazgos ecográficos asociados a una mayor probabilidad de malignidad son la presencia de microcalcificaciones, la vascularización central y la hipoecogenicidad. En cambio, los asociados a un menor riesgo son la presencia de halo, la existencia de áreas de degeneración coloide y la heterogeneidad de los nódulos ${ }^{7-8}$.

Estudios recientes sugieren que la evolución de los métodos diagnósticos como la ecografía de alta resolución, pueden ser la causa del aumento de la incidencia de CDT, sin poder descartarse posibles influencias ambientales y vías moleculares aún en estudio 9 .

Sin embargo, existe una limitación inherente a los estudios de imágenes debido a que es un método operador dependiente ${ }^{10}$.

\section{Objetivo Primario}

Determinar la precisión diagnóstica de los criterios ultrasonográficos sospechosos de malignidad de nódulos tiroideos para el diagnóstico de cáncer de tiroides tomando como estándar de oro el resultado anatomopatológico.

\section{Objetivo Secundario}

Determinar el valor de corte del número de criterios ecográficos de sospecha de malignidad que muestre el mejor balance de sensibilidad/especificidad para diagnosticar cáncer de tiroides.

\section{Pacientes Y Métodos}

Se realizó un estudio observacional, retrospectivo, de pruebas diagnósticas en el que se incluyeron pacientes adultos con nódulos sospechosos de malignidad por ecografía y punción aspirativa. En estos pacientes se evaluó la sensibilidad, especificidad, valores predictivos positivo $\mathrm{y}$ negativo de la presencia de al menos un criterio ecográfico positivo para malignidad tomando como gold standard el resultado anatomopatológico. Igualmente, se analizó el valor de corte del número de criterios ecográficos que mostrara la mejor relación de sensibilidad/especificidad.

Se analizaron las historias clínicas de pacientes adultos de ambos sexos intervenidos mediante cirugía tiroidea entre el $1^{\circ}$ de enero de 2012 y el 31 de diciembre de 2018 en la Clínica Universitaria Reina Fabiola en la Ciudad de Córdoba.

\section{Criterios de exclusión}

- Personas que no fueran pacientes del Servicio de Endocrinología de la CURF.

- Pacientes en los que no dispusiéramos con al menos una ecografía tiroidea prequirúrgica.

- Pacientes sometidos a tiroidectomía por hipertiroidismo.

- Pacientes con diagnóstico anatomopatológico final maligno, tras intervención de tiroidectomía, diferente a cáncer diferenciado de tiroides.

\section{Variables a consignar}

1) Edad

2) Sexo

3)Criterios ecográficos sospechosos de malignidad de nódulos tiroideos:
a) Hipoecogénico: SI, NO
b) Más alto que ancho: SI, NO
c) Microcalcificaciones: SI, NO
d) Bordes poco definidos: SI, NO
e) Invasión perinodular: SI, NO
f) Flujo Doppler central: SI, NO
g) Adenopatías: SI, NO

4) Anatomía patológica de pieza quirúrgica de tiroidectomía:

a) Maligno: cáncer de tiroides.

b) Benigno

5) Tipos de cáncer diferenciado de tiroides ${ }^{3}$ :

a) Carcinoma papilar: clásico, variante folicular, microcarcinoma papilar, encapsulada, células altas, oxifílica, esclerosante difusa, células columnares.

b) Carcinoma folicular: con mínima invasión capsular, amplia invasión capsular, encapsulado con invasión vascular. 
c) Carcinoma de células de Hurthle

\section{Tamaño muestral}

Para una sensibilidad de los criterios ecográficos sospechosos de malignidad para el diagnóstico de cáncer de tiroides del $88 \%$, un nivel de fiabilidad del $95 \%$ y un error del alfa del $5 \%$, el tamaño muestral se calculó en 50 .

\section{Recolección de datos}

Se ingresaron los datos de los pacientes y la información de su historia clínica en un documento de Microsoft Excel® y luego fueron analizados en el programa SPSS1 23.0 para Windows.

Para detectar los pacientes consecutivos intervenidos mediante cirugía tiroidea se realizó una búsqueda mediante el sistema informatizado del Servicio de Anatomía Patológica de la CURF.

\section{Análisis estadístico}

Los resultados se expresaron como porcentajes para variables categóricas, y como media y desviación estándar para variables continuas, utilizando la mediana y el rango intercuartílico para variables con distribución asimétrica. Se calcularon los parámetros de prueba diagnóstica sensibilidad, especificidad, valores predictivos (valor predictivo positivo [VPP], valor predictivo negativo [VPN]) de cada parámetro ecográfico y para la sumatoria de los mismos utilizando tablas de $2 \times 2$.

Para determinar el valor de corte del número de criterios ecográficos de sospecha de malignidad que muestre el mejor balance de sensibilidad/especificidad para diagnosticar cáncer de tiroides se calculó la media.

El análisis estadístico se realizó mediante el programa SPSS1 23.0 para Windows.

\section{Resultados}

Se revisaron 395 historias clínicas de pacientes. De ellos, se excluyeron $150(37,97 \%)$ por no disponer de seguimiento clínico, $10(2,53 \%)$ por no disponer de ecografía tiroidea previa a la cirugía, $10(2,53 \%)$ por haber sido operados por hipertiroidismo y los últimos $2(0,5 \%)$, uno debido a que presentó como diagnóstico final de su tiroidectomía una metástasis de tumor oculto que resultó cáncer de colon y el otro por resultar su pieza quirúrgica de tiroidectomía una histiocitosis tiroidea siendo ésta la primera manifestación de histiocitosis sistémica. Por lo tanto, se incluyeron los datos de 223 pacientes (56,45\%). De ellos 179 $(80,26 \%)$ eran de sexo femenino, con una edad media (desviación estándar - DE) de 43,49 (13,03) años.

Entre los pacientes con patología benigna, 65 casos $(52,42 \%)$ resultaron en adenoma folicular, habiendo representado el hallazgo anatomopatológico más frecuente (Tabla 1). Noventa y nueve $(44,39 \%)$ pacientes presentaron diagnóstico de cáncer de tiroides, siendo la totalidad de los mismos clasificados como carcinoma diferenciado de tiroides. Dentro de estos, $76(76.81 \%)$ eran de sexo femenino con una edad media (DE) de 40,36 $(12,48)$ años. La media (DE) del tamaño tumoral fue 20,35 $(12,73) \mathrm{mm}$. El $94,45 \%$ resultaron CDT papilar cuya variante más frecuente fue la clásica con 72 de 94 casos $(72,73 \%)$ (Tabla 1$)$

Tabla 1. Descripción anatomopatológica de los tumores tiroideos.

\begin{tabular}{|c|c|c|}
\hline TAMAÑO DE TUMORES $(\mathrm{mm})$ & Media & $\begin{array}{l}\text { Desviación } \\
\text { estándar }\end{array}$ \\
\hline Benigno & 23,62 & 14,34 \\
\hline Maligno & 20,35 & 12,73 \\
\hline DIAGNÓSTICO ANATOMOPATOLÓGICO & $\mathbf{N}$ & $\%$ \\
\hline Adenoma Folicular & 65 & 52,42 \\
\hline Bocio nodular macromicrofolicular & 39 & 31,45 \\
\hline Adenoma de células de Hurtle & 11 & 8,87 \\
\hline Hiperplasia folicular & 5 & 4,03 \\
\hline Adenoma trabecular hialinizante & 2 & 1,61 \\
\hline Tiroiditis de Riedel & 1 & 0,81 \\
\hline $\begin{array}{l}\text { Tumor bien diferenciado de potencial maligno } \\
\text { incierto }\end{array}$ & 1 & 0,81 \\
\hline Total & 124 & \\
\hline CARCINOMAS TIROIDEOS & $\mathbf{N}$ & Porcentaje (\%) \\
\hline Carcinoma papilar & 94 & 94,95 \\
\hline -Variante clásico & 72 & 72,73 \\
\hline - Variante folicular & 18 & 18,18 \\
\hline - Variante células altas & 2 & 2,02 \\
\hline - Variante oncocitica & 1 & 1,01 \\
\hline -Variante células columnares & 1 & 1,01 \\
\hline Carcinoma de células de Hurtle & 5 & 5,05 \\
\hline Total de carcinomas tiroideos & 99 & \\
\hline
\end{tabular}

\section{Análisis de los Criterios ecográficos sospechosos de malignidad}

La media (DE) de criterios ecográficos sospechosos en el grupo de pacientes con malignidad fue de 2,10 $(1,22)$, para el grupo de pacientes con resultado benigno fue de 1,36 $(1,08)$ ( $\mathrm{p}<0,001)$ (Figura 1).

La sensibilidad de los criterios ecográficos sospechosos de malignidad para el diagnóstico de cáncer diferenciado de tiroides fue superior en el criterio hipoecogénico con el $70,7 \%$, y la especificidad fue alta en los siguientes criterios: más alto que ancho, microcalcificaciones, bordes irregulares, invasión perinodular y adenopatías. Estos datos se muestran en la (tabla 2). 


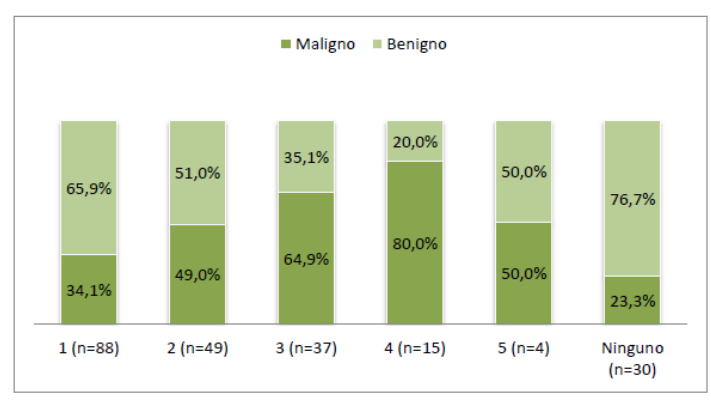

Figura 1. Número de criterios ecográficos de sospecha de malignidad según el resultado final de la tiroidectomía

Tabla 2: Sensibilidad, especificidad, valores predictivos positivos y negativos de los criterios ecográficos sospechosos de malignidad.

\begin{tabular}{|c|c|c|c|c|c|c|c|}
\hline \multirow[t]{2}{*}{ Indicadores } & \multicolumn{7}{|c|}{ Criterios Ecográficos } \\
\hline & (1) & (2) & (3) & (4) & (5) & (6) & (7) \\
\hline $\begin{array}{l}\text { Pacientes correctamente } \\
\text { diagnosticados } \\
\text { (coincidencias) }\end{array}$ & $\begin{array}{l}56,0 \\
\%\end{array}$ & $\begin{array}{l}57,4 \\
\%\end{array}$ & $\begin{array}{l}64,1 \\
\%\end{array}$ & $\begin{array}{l}61,0 \\
\%\end{array}$ & $\begin{array}{l}56,5 \\
\%\end{array}$ & $\begin{array}{l}50,7 \\
\%\end{array}$ & $\begin{array}{l}61,0 \\
\%\end{array}$ \\
\hline Sensibilidad & $\begin{array}{l}70,7 \\
\%\end{array}$ & $9,1 \%$ & $\begin{array}{l}42,4 \\
\%\end{array}$ & $\begin{array}{l}26,3 \\
\%\end{array}$ & $3,0 \%$ & $\begin{array}{l}44,4 \\
\%\end{array}$ & $\begin{array}{l}14,1 \\
\%\end{array}$ \\
\hline Especificidad & $\begin{array}{l}44,4 \\
\%\end{array}$ & $\begin{array}{l}95,9 \\
\%\end{array}$ & $\begin{array}{l}81,5 \\
\%\end{array}$ & $\begin{array}{l}88,7 \\
\%\end{array}$ & $\begin{array}{l}99,2 \\
\%\end{array}$ & $\begin{array}{l}55,6 \\
\%\end{array}$ & $\begin{array}{l}98,4 \\
\%\end{array}$ \\
\hline $\begin{array}{l}\text { Valor predictivo positivo } \\
\text { (VPP) }\end{array}$ & $\begin{array}{l}50,4 \\
\%\end{array}$ & $\begin{array}{l}64,3 \\
\%\end{array}$ & $\begin{array}{l}64,6 \\
\%\end{array}$ & $\begin{array}{l}65,0 \\
\%\end{array}$ & $\begin{array}{l}75,0 \\
\%\end{array}$ & $\begin{array}{l}44,4 \\
\%\end{array}$ & $\begin{array}{l}87,5 \\
\%\end{array}$ \\
\hline $\begin{array}{l}\text { Valor predictivo negativo } \\
\text { (VPN) }\end{array}$ & $\begin{array}{l}65,5 \\
\%\end{array}$ & $\begin{array}{l}56,9 \\
\%\end{array}$ & $\begin{array}{l}63,9 \\
\%\end{array}$ & $\begin{array}{l}60,1 \\
\%\end{array}$ & $\begin{array}{l}56,2 \\
\%\end{array}$ & $\begin{array}{l}55,6 \\
\%\end{array}$ & $\begin{array}{l}58,9 \\
\%\end{array}$ \\
\hline
\end{tabular}

\section{Discusión}

Varias características ecográficas han sido asociadas con un riesgo incrementado de cáncer de tiroides, incluida la presencia de calcificaciones, hipoecogenicidad, márgenes irregulares, ausencia de halo, componente predominantemente sólido, y vascularización intranodular, acorde a lo descripto en el estudio TIRADS ${ }^{19}$.

Nuestros hallazgos son congruentes con lo establecido por la ATA en su guía sobre el manejo de nódulos tiroideos y cáncer diferenciado de tiroides, donde describe que los criterios ecográficos que se asocian con cáncer de tiroides son las microcalcificaciones, bordes irregulares, más alto que ancho e hipoecogenicidad, siendo las tres primeras las de mayor especificidad $(>90 \%)$ y las que, asociadas a un nódulo sólido, tienen el mayor riesgo estimado de malignidad (>70$90 \%)^{10}$.

La Asociación Europea de Tiroides en la guía EUTirads reporta que los criterios ecográficos sospechosos de malignidad tienen sensibilidad y especificidad variables, pero que ninguno de ellos por si solo es suficiente para descartar o detectar malignidad eficientemente. Establece un grupo de alto riesgo conformado por una de las características ecográficas con elevada sospecha: bordes irregulares, microcalcificaciones, hipoecogenicidad $(26-87 \%$ de riesgo de malignidad). Además, resalta la importancia de la variación interobservador en la realización de las ecografías $^{21}$.

La Sociedad Francesa de Endocrinología en su guía para el manejo de nódulo tiroideo describe que las microcalcificaciones, bordes irregulares, hipoecogenicidad se asocian con un elevado riesgo de malignidad ${ }^{20}$.

Iannuccilli et al. en su estudio "Risk for Malignancy of Thyroid Nodules as Assessed by Sonographic Criteria" afirman que las microcalcificaciones son el único criterio estadísticamente significativo ${ }^{15}$ y Papini et al. en "Risk of Malignancy in Nonpalpable Thyroid Nodules: Predictive Value of Ultrasound and Color-Doppler Features" encuentran que los hallazgos ecográficos que tienen poder predictivo de malignidad son el patrón vascular intranodular, las microcalcificaciones y los márgenes irregulares ${ }^{16}$.

Kwak et al, por su parte, hallan una asociación significativa de malignidad con la presencia de componente sólido, microcalcificaciones, márgenes irregulares o microlobulados, hipoecogenicidad y más alto que ancho ${ }^{17}$.

Frates et al refieren que el hallazgo ecográfico con mayor sensibilidad es la composición sólida del nódulo, pero con un VPP muy bajo, y que el hallazgo con mayor VPP son las microcalcificaciones ${ }^{18}$.

En nuestro análisis las adenopatías tuvieron el mayor VPP correspondiéndose con el mayor índice de malignidad. Cabe destacar a partir de esto, que los estudios reportados no incluyen a las adenopatías como criterio ecográfico de sospecha; sin embargo, la ATA en su guía afirma la importancia de la búsqueda de las mismas en la ecografía ante la evaluación de un nódulo tiroideo $^{10}$, así como la Sociedad Francesa de Endocrinología en su guía recomienda la detección de ganglios sospechosos ultrasonográficamente ${ }^{20}$. Nuestros resultados establecen que a mayor número de criterios ecográficos sospechosos (con un mínimo de dos), aumenta la probabilidad de que ese nódulo sea maligno. Con respecto a esto, Uliaque et al sostiene que es necesaria la presencia conjunta de varios hallazgos ecográficos para realizar un mejor diagnóstico de la malignidad de un nódulo tiroideo ${ }^{4}$. También la Sociedad Francesa de Endocrinología en su guía afirma que cuando se combinan estas variables, el riesgo de malignidad aumenta significativamente (VPP $75 \%)^{20}$, y la ATA en su guía reporta que las sensibilidades son significativamente más bajas para cada una por separado $^{10}$.

El EU-Tirads recomienda que los nódulos que tienen al menos una característica ecográfica sospechosa de malignidad deben ser considerados con alto riesgo de malignidad, incrementándose el riesgo con el número de criterios ${ }^{21}$. En un extenso 
estudio coreano que evaluó más de 1600 pacientes que representó la base para la creación del score TIRADS $\mathrm{Kwak}^{22}$. Asimismo, en un análisis retrospectivo realizado en un centro austriaco se determinó como punto de corte para predecir malignidad la presencia de dos criterios, con una sensibilidad del $85 \%$ y una especificidad del $45 \%{ }^{7}$. En nuestro trabajo cabe mencionar que nuestras limitaciones incluyen acorde a lo descripto en la bibliografía, la variabilidad interoperador en la realización de las ecografías ${ }^{10}$. Por otro lado, nuestra población estaba conformada por pocos pacientes que tuvieran cinco criterios ecográficos sospechosos y ninguno con seis, por lo que en esos grupos la muestra no era representativa para realizar un análisis.

\section{Conclusión}

La ecografía es el método inicial de screening de los nódulos tiroideos. Si bien algunos criterios ecográficos tienen mayor sensibilidad para el diagnóstico de cáncer de tiroides que otros, la precisión diagnóstica del método se incrementa a medida que aumenta el número de características sospechosas detectados.

Este estudio permitirá conocer en que pacientes debe considerarse realizar tiroidectomía acorde a la probabilidad de cáncer de tiroides descripta según los criterios de malignidad ecográficos.

\section{Bibliografía}

1. Franco Uliaque C, Pardo Berdún F, Laborda Herrero R, Lórenz C. Utilidad de la ecografía en la evaluación de los nódulos tiroideos. Radiología. 2019; 58 (5):380-388.

2. Reverter J, Colomé E, Halperin I, Julián T, Díaz G, Mora M et al. Estudio comparativo de las series históricas de carcinoma diferenciado de tiroides en dos centros hospitalarios de tercer nivel españoles en relación a series norteamericanas. Endocrinología y Nutrición. 2010; 57(8):364-369.

3. Pitoia F. Carcinoma diferenciado de Tiroides. Primera Parte. Separata. 2014; 22(2):1-36.

4. Pitoia F. et al. Consenso intersocietario sobre tratamiento y seguimiento de pacientes con cáncer diferenciado de tiroides. RAEM. 2014; 51(2):85-118.

5. Cabezón C. Carcinoma diferenciado de Tiroides. Segunda parte. Separata. 2014; 22(4):1-36.

6. Horvath E, Clasificación TIRADS. Una herramienta útil en la selección de nódulos tiroideos que requieren punción diagnóstica. Contacto Científico. 2015; 2 (5).

7. Tugendsam C, Petz V, Buchinger W, Schmoll-Hauer B, Schenk I, Rudolph K et al. Ultrasound criteria for risk stratification of thyroid nodules in the previously iodine deficient area of Austria - a single centre, retrospective analysis. Thyroid Research. 2018; 11(3):1-8.

8. Tessler F, Middleton W, Grant E, Hoang J. Re: ACR Thyroid Imaging, Reporting and Data System (TI-RADS): White Paper of the ACR TI-RADS Committee. Journal of the American College of Radiology. 2018; 15(3):381-382.

9. Haugen B, Alexander E, Bible K, Doherty G, Mandel S, Nikiforov Y et al. 2015 American Thyroid Association Management Guidelines for Adult Patients with Thyroid Nodules and Differentiated Thyroid Cancer: The American Thyroid Association Guidelines Task Force on Thyroid Nodules and Differentiated Thyroid Cancer. Thyroid. 2016; 26(1):1-133.

10. Mora-Guzmán I, Muñoz de Nova J, MarínCampos C, Jiménez-Heffernan J, Cuesta Pérez J, Lahera Vargas M et al. Rendimiento del sistema Bethesda en el diagnóstico citopatológico del nódulo tiroideo. Cirugía Española. 2018; 96(6):363-368.

11. Romero-Rojas A, Melo-Uribe M. Implementación del Sistema Bethesda para el informe de citología aspirativa de tiroides con seguimiento histopatológico: experiencia en un centro de tratamiento de cáncer. Revista Colombiana de Cancerología. 2014; 18(1):3-

12. Mileva M, Stoilovska B, Jovanovska A, Ugrinska A, Petrushevska G, KostadinovaKunovska $\mathrm{S}$ et al. Thyroid cancer detection rate and associated risk factors in patients with thyroid nodules classified as Bethesda category III. Radiology and Oncology. 2018; 52(4):370-376.

13. Fernández Sánchez J. Clasificación TI-RADS de los nódulos tiroideos en base a una escala de puntuación modificada con respecto a los criterios ecográficos de malignidad. Rev Argent Radiol. 2014; 78(3):138-148.

14. Casella C, Ministrini S, Galani A, Mastriale F, Cappelli C, Portolani N. The New TNM Staging System for Thyroid Cancer and the Risk of Disease Downstaging. Front Endocrinol (Lausanne). 2018; 9(541):1-4.

15. Iannuccilli J, Cronan J, Monchik J. Risk for Malignancy of Thyroid Nodules as Assessed 
by Sonographic Criteria. Journal of Ultrasound in Medicine. 2004;23(11):14551464.

16. Papini E. Risk of Malignancy in Nonpalpable Thyroid Nodules: Predictive Value of Ultrasound and Color-Doppler Features. Journal of Clinical Endocrinology \& Metabolism. 2002;87(5):1941-1946.

17. Kwak J, Han K, Yoon J, Moon H, Son E, Park $\mathrm{S}$ et al. Thyroid Imaging Reporting and Data System for US Features of Nodules: A Step in Establishing Better Stratification of Cancer Risk. Radiology. 2011;260(3):892-899.

18. Fahey T. Management of Thyroid Nodules Detected at US: Society of Radiologists in Ultrasound Consensus Conference Statement. Yearbook of Surgery. 2006; 2006:146-148.

19. Horvath E, Majlis S, Rossi R, Franco C, Niedmann J, Castro A et al. An Ultrasonogram Reporting System for Thyroid Nodules Stratifying Cancer Risk for Clinical Management. The Journal of Clinical Endocrinology \& Metabolism. 2009;94(5):1748-1751.

20. Wémeau J, Sadoul J, d'Herbomez M, Monpeyssen H, Tramalloni J, Leteurtre E et al. Guidelines of the French society of endocrinology for the management of thyroid nodules. Annales d'Endocrinologie. 2011;72(4):251-281.

21. Russ G, Bonnema S, Erdogan M, Durante C, Ngu R, Leenhardt L. European Thyroid Association Guidelines for Ultrasound Malignancy Risk Stratification of Thyroid Nodules in Adults: The EU-TIRADS. European Thyroid Journal. 2017;6(5):225237.
22. Gharib H, Papini E, Paschke R, Duick D, Valcavi R, Hegedüs L, \& Vitti P. American Association of Clinical Endocrinologists, Associazione Medici Endocrinologi, and European Thyroid Association Medical Guidelines for Clinical Practice for the Diagnosis and Management of Thyroid Nodules: Executive Summary of Recommendations. Endocrine Practice. 2010;16(3):468-475.

23. Moon W, Jung S, Lee J, Na D, Baek J, Lee Y, Lee D. Benign and Malignant Thyroid Nodules: US Differentiation-Multicenter Retrospective Study. Radiology. 2008; 247(3):762-770.

24. Cameselle-Teijeiro $\mathbf{J}$ et al. Nueva clasificación de la OMS de los tumores tiroideos: una categorización pragmática de las neoplasias de la glándula tiroides. Elsevier. 2018; 65(3):133-135.

25. Park S, Oh C, Cho H, Lee J, Jung K, Jun J et al. Association between screening and the thyroid cancer "epidemic" in South Korea: evidence from a nationwide study. BMJ. 2016; 5745.

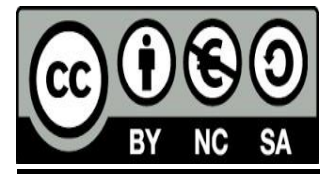

